\title{
"SmartGlass" Obstacles for Dynamic Inducing of Light Scattering in Vision Research Experiments
}

\author{
Olga DANILENKO ${ }^{1,2}{ }^{*}$, Maris OZOLINSH ${ }^{1}$, Varis KARITANS ${ }^{1}$, Paulis PAULINS ${ }^{1}$ \\ ${ }^{1}$ Institute of Solid State Physics, University of Latvia, 8 Kengaraga Str., Riga, LV-1063, Latvia \\ ${ }^{2}$ Department of Optometry and Vision Science, University of Latvia, 1 Jelgavas Str., Riga, LV-1004, Latvia \\ cross $^{\text {ref }}$ http://dx.doi.org/10.5755/j01.ms.22.4.12907
}

Received 19 August 2015; accepted 19 December 2015

\begin{abstract}
We describe a technique that allows control of visual stimuli quality through the use of a setup with a polymer dispersed liquid crystal (PDLC) film positioned in the optical pathway of one or both human eyes. Nowadays, PDLC films allow alteration of the resolution and contrast limits of the transmitted light due to continuous change in the light scattering that is obtained by the application of an AC electrical field. In our experimental setup, the use of a wide-aperture up to area of $20 \times 15 \mathrm{~cm}^{2}$ PDLC sheet is combined with a flat-screen PC display or with a modified display emission block without its interference filter unit and with an installed individually controllable colored light-emitting diode (LED) backlight. In the latter case, the spatial structure of visual stimulus remains constant, but the PDLC switching-on timing for intensity, color, and contrast of visual stimuli control is done by a PC via an Arduino USB interface. Arduino applies a voltage to the backlight colored LEDs and the low voltage up to $30-80 \mathrm{~V}$ to light-scattering PDLC sheet. Modifications to this setup can improve the resolution of the timing and screen stimulus intensity and color purity, and increase the flexibility of its application in visual research tasks. A particular use of PDLC scattering sheets involves the altering of the stimuli input strength of the eye in different binocular viewing schemes. In such applications, a restrictedoptical-aperture PDLC element is mounted in a goggle frame, and the element is controlled by the application of lowvoltage AC field. The efficacy of the setup is demonstrated in experiments of human vision contrast sensitivity adaptation studies. Studies allow to determine the characteristic time of the contrast sensitivity altering of $4 \mathrm{~s}$ during adaptation phase and the same order of the characteristic time during recovery.

Keywords: LED monitors, Arduino interfaces, polymer disperse liquid crystals, light scattering, visual perception.
\end{abstract}

\section{INTRODUCTION}

Advances in materials science promote studies in many fields of optics including vision research. Human eyes together with their conjugated "personal computer" neural cells of the retina and the brain cortex fulfil their duties well and in a very flexible way, although eye optical characteristics are far from perfect. Particular interest in vision research is focused on finding the specific contribution of neural and optical factors to altering of the precise evaluation of optical stimuli in our brain perception tasks. Speaking of main optical causes of image deterioration in eye, authors mentioned eye defocusing and other optical aberrations, and light scattering [1-3]. Various ways are used to find how the intraocular scattering changes the eye resolution and affects measurements of eye aberrations [3-5].

Researchers have developed several visual optical models to study and objectively measure the impact of light scattering using various conventional materials. Previous studies [5] have reported on the development of a model eye with an element filled with lipid solution that affords good scattering control. However, the proposed device has a slow operation time, and it can be impractical for application in visual perception studies. Other researchers have considered suitable glass obstacles with calibrated scattering characteristics to simulate an eye

\footnotetext{
* Corresponding author. Tel.: +371-67260868; fax: +371-67132778.

E-mail address: olgadanilenko941@gmail.com (O. Danilenko)
}

suffering from cataract [6, 7]. However, such approaches require mechanical control to alter the degree of scattering, and hence, they do not provide lasting solutions. In this context, researchers are now focusing on "smart" materials that enable suitable variation in the optical properties of devices for various applications [8-11].

As the first example of the use of electrically controllable light scattering for eye cataract modeling, a study has reported on the use of lanthanum-modified lead zirconate titanate (PLZT) electro-optic ceramics [10]. This material allows "smart" variation in the device optical properties via external control in several ways, for example, by the application of an electrical field or based on the intensity of incident light $[12,13]$. However, the material requires a high operational electrical control voltage of up to $1000 \mathrm{~V}$. Other studies have reported on the use of layers of polymer dispersed liquid crystal (PDLC) embedded between two glass or plastic sheets covered with sputtered electroconductive indium tin oxide (ITO) as an attractive alternative $[8,9,14-16]$. The primary advantage of the PDLC over related materials is that it requires low control voltages $(30-80 \mathrm{~V})$, thereby allowing the implementation of easy-to-use devices in experiments on human vision perception. Other breakthroughs in materials science in this regard include the development of easily controllable portable light sources such as diode pumped solid state (DPSS) lasers and light emitting diodes (LEDs), which significantly simplify experiments [17, 18].

The setup proposed in this study qualitatively improves on conventional vision research techniques based 
on the demonstration of visual stimuli exhibited on a PC screen.

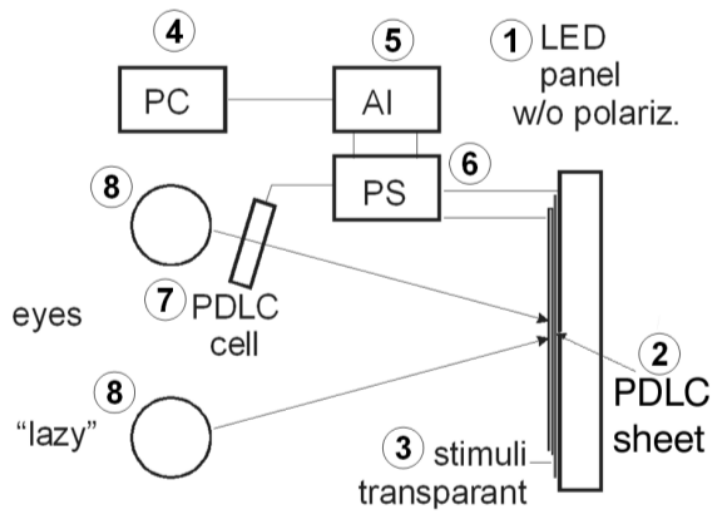

Fig. 1. Layout of visual perception experiments: $\mathrm{PC}-$ personal computer; $\mathrm{Al}$-Arduino interface board; $\mathrm{PS}$-power supply unit

Despite increases in CPU speeds, the visual output of conventional flat-screen displays remains time-quantized by the frame frequency, and this increases the complexity of achieving continuous timing of the demonstrated events.

The main aim of the present work is the development of a modified experimental technique that allows inducing of fast and controllable light scattering of visual stimuli and its application in visual perception studies on contrast sensitivity adaptation.

\section{EXPERIMENTAL SETUP}

Composite images or specific gratings for human visual perception studies (Gabor gratings of a single spatial frequency and without sharp spatial luminance transients) were generated by means of a computer video card on a PC screen (Fig. 1) or using prints on transparencies attached to a flat light-emitting panel. In both cases including the latter solution, we used the "squared" 17-inch Benq BL702A monitor (1) with LED backlight (pixel pitch of $0.255 \mathrm{~mm}$ ) as the base. As a wide-aperture SmartLight light-scattering obstacle, a PDLC (2) layer [9] mounted between two plastic films was affixed to the screen in front of the visual stimulus (3). The size of the working area of the PDLC film was $A=20 \mathrm{~cm} \times 15 \mathrm{~cm}$. Applying an AC voltage of $U_{\mathrm{AC}}=80 \mathrm{~V}$ (frequency $f=1-3 \mathrm{kHz}$ ) switches the PDLC layer from the translucent state to the transparent state. The Benq BL702A monitor uses white LEDs for the backlight, and the resulting wide spectrum of emission decreases the colour gamut of the reproducible colours. Therefore, we replaced the conventional LEDs by individually addressed LED elements (on the base of a WS2812 LED strip) with elementary coloured emission source groups on different substrate media for RGB light emission. The background colour was adjusted by varying the current to the R-, G-, and B-group LEDs. Next, PC (4) control of the LED current was achieved with the use of the Arduino USB interface (5). Such a solution allows independent timing of the demonstrated visual stimuli with submillisecond resolution.

The same Arduino interface controls the voltage (up to $80 \mathrm{~V}$ ) of the PDLC SmartGlass obstacle via an electronic unit (6) to achieve variation in the degree of light scattering and contrast of stimulus transparency (Fig. 2). As the controlled light scattering aperture $(20 \mathrm{~cm} \times 15 \mathrm{~cm})$ is smaller than the display area, only the central part of the LCD was used to display visual stimuli. The video output of other screen pixels was nulled. The screen luminance in this central part as measured by the MinoltaCS100A when adjusted at $250 \mathrm{~cd} / \mathrm{m}^{2}$ had a standard deviation of $S D=1.3 \mathrm{~cd} / \mathrm{m}^{2}$.

In the case when dynamic changes in the stimulus contrast require to be applied to one eye only, a smalleraperture PDLC cell (7) is inserted in a special frame that is placed in front of the corresponding eye. The PDLC layer of such an obstacle is positioned between two glass plates covered with transparent ITO electrodes. The schematic of the electro-optic operation of the dispersed light scatters in the polymer media under an applied electric field is depicted in Fig. 2. In absence of the electric field, the directors of individual microdroplets are randomly distributed in space, but the application of the electric field aligns their directors along the field. The refractive indices for the light beam in the polymer and droplets equalize when the light beam passes through the media along the optical axes of the liquid crystal droplets. Consequently, scattering in such a case is reduced to a minimum. Fig. 2 also shows the transmittance variation in the PDLC cell with working aperture $D=25 \mathrm{~mm}$ (bottom panel) when the electric field is present or absent. The cell aperture size is considerably larger than the eye's iris opening $(2-7 \mathrm{~mm})$.

The Arduino USB interface is available in a number of versions [19]. It is simple to use and allows control of the different elements involved in the experiment (electromechanical actuators, motors, optical, or RF distance controls). This control can be realized along with control and timing of the electrical analogue, pulse-width modulation (PWM), and acquisition of signals by the builtin microprocessor. Internet blogs on Arduino boards provide users with open codes that are applicable to many electromechanical and optoelectric devices available in the market.
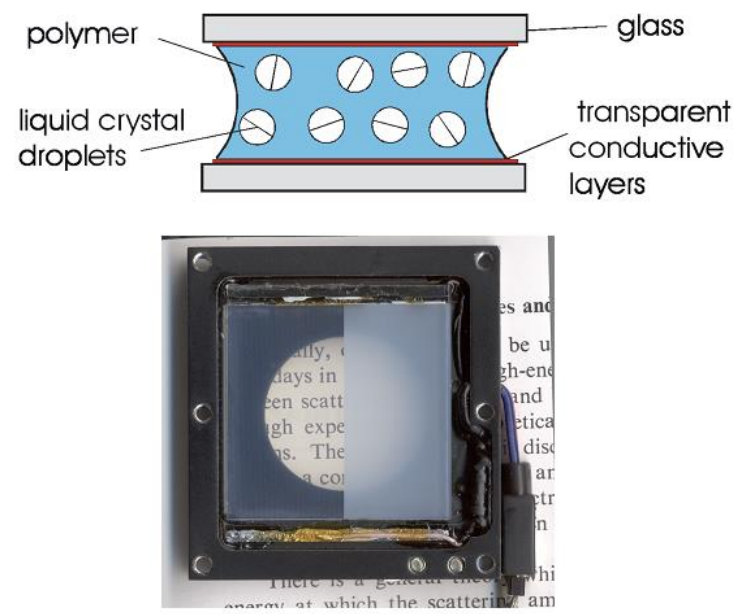

Fig. 2. Schematics of polymer dispersed liquid crystal PDLC layer placed in between two glass plates (top panel). Design of the frame with PDLC cell to achieve electrically controlled light scattering (bottom panel). The left side of the photomontage is visible when an AC voltage applied and the right side is visible in the absence of the voltage 


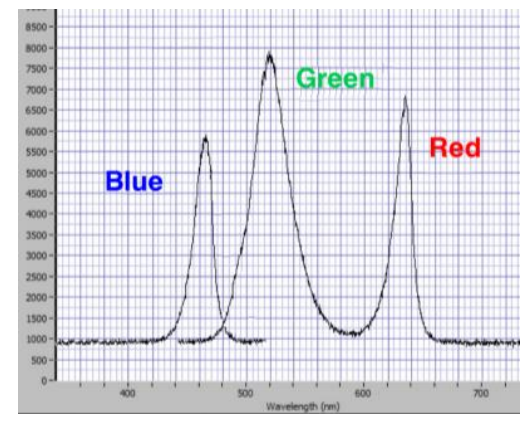

a

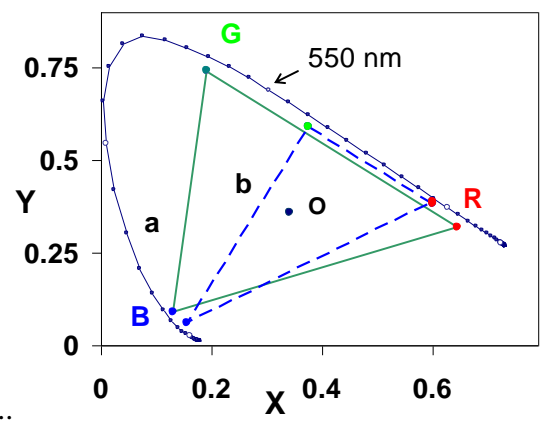

b

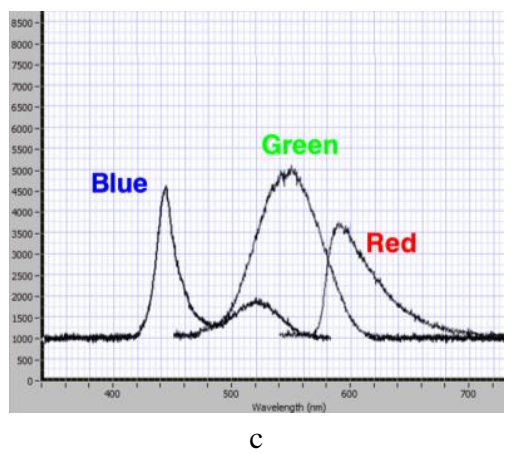

c

Fig. 3. a-emission spectra of panel with the modified set of R, G and B LEDs; $b$-emission loci in the CIE XY ((a) for modified an (b) for conventional LEDs backlight) chart for both the abovementioned cases $\mathrm{c}$-emission spectra of panel with built-in "white" LED backlight

\section{INVESTIGATION OF OPTICAL CHARACTERISTICS OF SETUP}

The spectral emission of the monitor panel (depicted in Fig. 3) before and after replacement of the built-in backlight by R, G, and B LEDs was investigated with the use of an Ocean Optics fiber-optic spectrometer. Further, the luminance homogeneity across the screen and the panel color locus in CIE XY coordinates were measured with a MinoltaCS100A chromameter. From Fig. 3, we observe that the modification of the backlight yields an improvement of the locus area in the CIE XY chart and of the screen colour purity. The main benefit of using R, G, and B LEDs is the increase in operational flexibility in terms of the device's independence of the computer video card timing of the visual stimuli demonstration. The luminance of the "white" could be easily stabilized at levels up to $250 \mathrm{~cd} / \mathrm{m}^{2}$ by appropriately adjusting the LED current.

Next, the effectiveness and time dependences of continuous light scattering were studied via application of an electric field to the PDLC sheet. A steady sinusoidal unidirectional grating with a spatial frequency of $f_{s}=3$ cycles/degree was generated on the monitor with the monitor Videosignal/Luminance gamma [20] function $\gamma=1$ and photographed with a conventional LumixGF1 camera for different $\mathrm{AC}$ voltages $(f=3 \mathrm{kHz})$ applied to the wide-aperture PDLC sheet. The resulting decreases in the estimated stimuli contrast and the mean luminance are shown in Fig. 4.

Similar measurements were performed for the "smallaperture" PDLC cell via the same technique. In this case, the PDLC cell was located in front of the image formatting optics (human eye or camera lens f 2.8 Olympus OM with focal length of $24 \mathrm{~mm}$ ). The voltage required to switch the PDLC cell to the transparent state was correspondingly lower (Fig. 5) than for cells of related electro-optic material PLZT [11] ceramics which however are considerably faster than PDLC even allowing to realize laser Q-switching [21] but having $\mathrm{kV}$-level control voltages. The voltage required to attain maximum transparency for the small-aperture PDLC cell was $U=30 \mathrm{~V}$. The Scattering(contrast)/Voltage characteristics for the cases of large-area or small-area cells are definitely nonlinear.

In both experimental schemes, the reduction of contrast can be measured photographically. We chose the human observer viewing distance as $d=0.7 \mathrm{~m}$, and the same distance was used in experiments for objective photometrical calibration of the stimuli grating contrast transfer. As reference objects, the photocopies of the gradual step function and Gabor gratings with Michelson contrast $C_{M}$ were utilized:

$C_{M}=\frac{L_{\text {max }}-L_{\text {min }}}{L_{\text {max }}+L_{\text {min }}}$,

where $L_{\max }$ and $L_{\min }$ represent the luminances of the maxima and minima in the Gabor stimuli, respectively.

The aperture and exposure settings of the camera were kept unchanged via the use of the camera's manual control mode in all measurements. The complex photometric $\gamma$-function impact in calibration was determined by parallel measurement of the object segment luminance by means of the MinoltaCS100A chromameter. The determined pixel RGB values were used as the raw data for calculations of stimuli contrast taking into account the calibration.

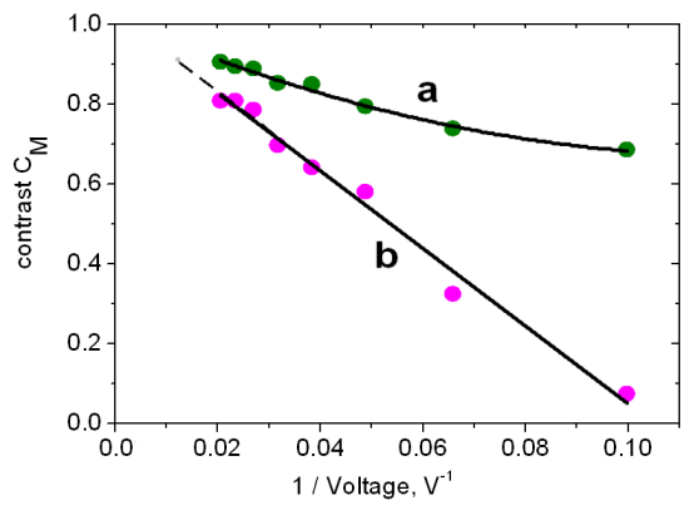

Fig. 4. Variation in the relative mean screen-luminance (a) and the contrast of the unidirectional sinusoidal grating stimulus (b) when an AC voltage is applied to the wideaperture PDLC cell (black-white grating spatial frequency $f_{s}=3$ cycles/deg) 


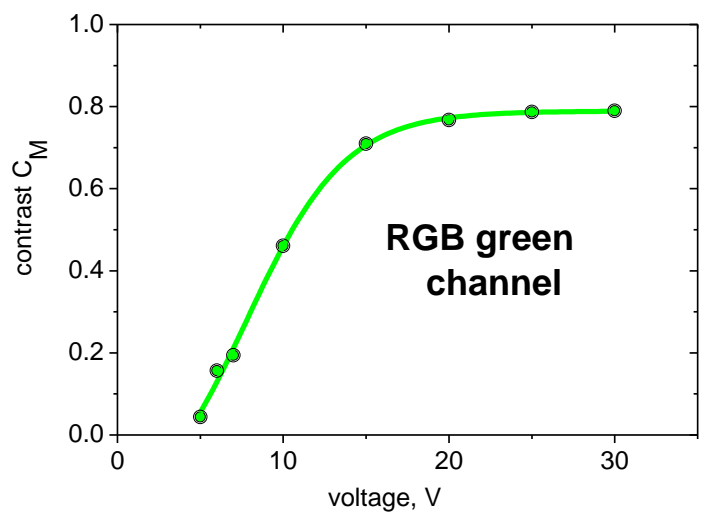

Fig. 5. Variation in the contrast of the line grating stimulus photograph through PDLC cell when an AC voltage is applied to the small-aperture light-scattering PDLC cell (photograph green channel, grating spatial frequency $f_{s}=3$ cycles $/ \mathrm{deg}$ )

However, the scattering-induced reduction in the spatial resolution of the stimuli over the range of human visual acuity was difficult to evaluate. The only suitable experimentally available spatially sensitive indicator was the human eye.

With the use of the human eye as the indicator of changes in spatial resolution we observed, that the scattering that causes the impact on the perceived stimuli contrast is, at least at sufficient luminance levels, a secondorder effect in eye spatial resolution. The main cause of blurring still is due to defocusing and wide spectrum of optical aberrations $[1,2,6]$. However, the latter effect also can be introduced in such experiments using electrically controllable liquid lenses [10].

\section{APPLICATION OF TECHNIQUE IN VISION PERCEPTION STUDIES}

We applied the proposed technique in studies of human vision adaptation (young optometry students with normal corrected decimal visual acuity not less than 1.0 were as observers) and perceived stimuli contrast amplification in the brain structures. To improve visual perception and dynamical range, the vision biochemistry in humans utilizes feedbacks that also allow alteration of the limits of the input contrast range. This process can occur in an interval of a few seconds. We briefly examine certain aspects of the process here. First, this kind of adaptation is based on certain processes occurring in retinal neural cells and is thus also determined by monocular vision. Secondly, information processing in the cells of the visual cortex is also determined by vision binocularity. Here, we remark that it is difficult to directly measure visual contrast adaptation. One possible approach involves stroboscopic probing of the neural processing with the application of repeatable visual stimuli. Here, the sequence of events in the experimental protocol should be organized suitably, since the adaptation process occurs in a timescale of seconds and milliseconds.

The next problem in these studies is the strength of the vision contrast amplification adaptation effect. This can depend on whether the inputs from one or both eyes participate or interact in the final YES/NO switching in the brain's decision-making process. Although the basis of all events lies in the field of cortex biochemistry, experimentally, it is possible to interact with them by controlling the optical content of both eye inputs. The solving of such experimental tasks can be improved by varying the timing and dynamic control of the visual stimuli optical contrast. In combined studies of binocular and monocular perception of contrast adaptation, we used a light-scattering PDLC obstacle over a timescale in the range up to $15 \mathrm{~s}$ (Fig. 6).

In these cases, when perception is binocular, we used a PC screen to display the black-white greyscale adapting stimuli that comprised Gabor gratings with a spatial frequency $f s$ and with different Michelson contrast values of the adaptation stimulus $C_{\mathrm{M}-\mathrm{AD}}$.

During the adaptation phase (interval of CA seconds in Fig. 6), the perceived contrast amplification in the observer's cortex diminishes. This phenomenon can be interpreted as the induction of a negative aftereffect that is added to the positive adaptation stimulus. The test stimulus appearing during the recovery phase, the phase of observation that begins immediately after the instant $t 1$, consists of "compound" areas-two upper and lower "sidebands" (shown as the top and bottom sections of the stimuli in Fig. 6) with constant high-contrast reference gratings (spatially in phase with the adapting stimulus). Furthermore, the test stimulus contains the central test area of the control (compensating) grating of contrast $C_{\mathrm{M}-\mathrm{T}}$ also as an in-phase grating. The contrast $C_{\mathrm{M}-\mathrm{T}}$ value randomly varies in subsequent trials. The alternative forced twochoice task paradigm was used to evaluate the psychophysical testing of vision. The observer's task was to answer the following two-part question:

"Is the perceived grating in the central test area inphase with the two upper and lower reference gratings? Or is it in counter-phase?"

Here, we remark that the answer is YES if the compensation stimuli contrast in the test area is over the contrast threshold. When the answer is NO, it indicates that the compensation stimulus contrast is below the threshold.

The observer responses were acquired by the pressing of the appropriate keyboard keys. Up to 10 random presentations were made per trial series with the stimuli of the fixed test grating contrast. YES responses were assigned a value of " 1 " and NO responses were assigned a value of " 0 ," and the responses were summed.

The resulting psychophysical curves were fitted to a sigmoid function, wherein the trial sample test stimulus contrast was used as the ordinate.

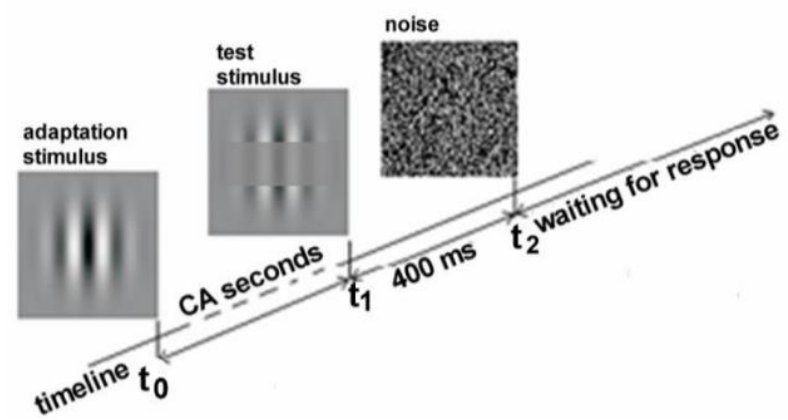

Fig. 6. Time sequence of eyes adaptation to stimulus contrast experiment 


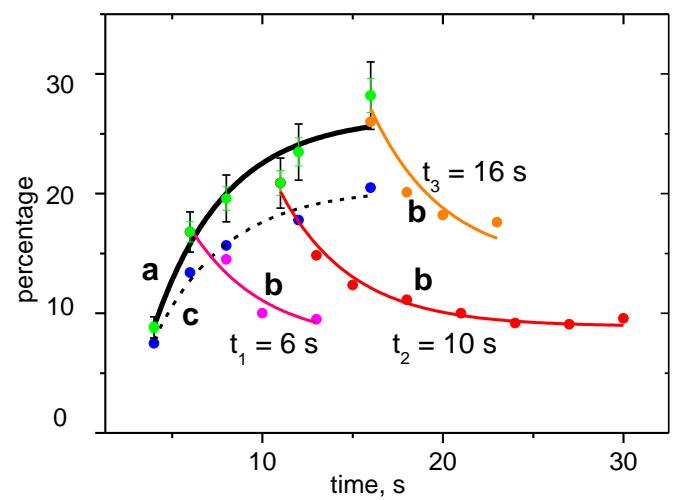

Fig. 7. Time courses during adaptation to the high-contrast Gabor grating with $C_{\mathrm{M}-\mathrm{AD}}=70 \%$ : (a) the time course of contribution of the adaptation part for binocular viewing $v s$. adaptation period (solid bold line); (b) the time courses of contribution of the value of the adaptation part for binocular viewing $v s$. time after switching off the adaptation stimulus at various $t 1$ values (solid lines); (c) the time course of contribution of the adaptation part for binocular viewing $v s$. adaptation period with one eye covered during the adaptation by PDLC cell which decreases the contrast to $50 \%$ level (dashed line)

At the fitted slope symmetry center, a compensation of the perception of the central part in-phase stimulus and the counter-phase afterimage grating occurs, wherein the probability of YES and NO responses equalizes. We used the PDLC cell partially to block high $C_{\mathrm{M}-\mathrm{AD}}$ adaptation stimuli for one eye during the adaptation phase to compare results of the cortex contrast amplification under different viewing conditions. Fig. 7 shows the estimated time dependencies during human vision contrast adaptation. The following measure here is understood as the determined value: the perceptual change (decrease) in the contrast amplification in the visual pathway after adaptation to the high-contrast adaptation grating. The time dependencies in Fig. 7 can be divided into three sections: (a) the time course corresponding to decrease in contrast amplification, which is the contribution of the adaptation part for binocular viewing vs. adaptation period (solid bold line); (b) the time course corresponding to decrease in contrast amplification, which is the contribution of the value of the adaptation part for binocular viewing vs. time after switching off the adaptation stimulus at various $t l$ values (solid lines); and (c) the time course corresponding to decrease in contrast amplification, which is the contribution of the adaptation part for binocular viewing vs. adaptation period with one eye covered during adaptation with the PDLC cell which decreases the contrast to $50 \%$ level (dashed line).

In the case of binocular viewing conditions with fixed view of sight at the stimulus center, the time courses for the perception contrast decrease follows an exponential law with the characteristic time $\tau \approx 4 \mathrm{~s}$. An exponential fit of the recovery of the contrast sensitivity shows the same value of the characteristic time.

\section{DISCUSSION AND CONCLUSIONS}

Modifying the screen backlight with the introduction of separately addressable LED light elements overcomes several conventional experimental restrictions. Computer displays can be exploited for vision studies both with the $\mathrm{PC}$ video output in the conventional manner or with a removable polarizer and LC panel. In such cases, steady visual and spatially organized stimuli content can be dynamically controlled using the screen backlight luminance control and dynamic contrast control via light scattering PDLC elements. In specific cases, this can simplify the stimuli demonstrations. PDLC sheets can be directly affixed as large-aperture light obstacles to the display polarizer sheet or on the surface of the specified steady visual stimuli transparency.

Such scattering control allows the demonstration of visual stimuli via the application of an AC voltage $U_{\mathrm{AC}}=0-80 \mathrm{~V}$ while continuously diminishing the Michelson contrast of Gabor gratings in range of $C_{\mathrm{M}}=0-80 \%$.

Our proposed device allowed the study of such visual characteristics as the adaptation of vision contrast sensitivity. The altering of the perception can be characterized by the contrast amplification characteristic time of $\tau \approx 4 \mathrm{~s}$ that is typical to enzymatic processes occurring at the retinal and cortical levels of visual perception. The contribution of faster adaptation processes, according to processing of our data, seems negligible. It is not possible to mark a certain "jump-like" increase in the time-course curves of the altering of visual stimuli contrast amplification in a time less than $1 \mathrm{~s}$. Curve fitting reveals the slight contribution of slower adaptation; however, our measurement protocol does not allow the determination of its time constant due to this contribution being almost linear in the time range (15 s) of our studies.

It is possible to assume that the adaptation occurs at the retinal level, most probably as the retinal aftereffect, and at the cortex level as a pure contrast sensitivity altering. In order to separate these contributions, one can perform measurements either with floating sight fixation during adaptation or by monocular viewing conditions (alternately covering one eye during adaptation and the other eye during recovery phases).

When there is a need to separate both eye inputs, a low-voltage PDLC element can be mounted in a frame placed directly in front of one eye. The Arduino type interface unit attached to the computer's USB port allows easy control of the display backlight and efficiency of light scattering provided by the PDLC element. The impressing of visual stimuli contrast plays a key role in studies involving certain perceptual tasks or pathologies such as eye cataract. In our study, we analyzed the use of dynamically switchable light scattering in studies of the peculiarities of the process of visual perception contrast adaptation, which depends on various visual perception stages, and on binocular or monocular neural processing. Finally, the availability of numerous types of actuators and sensors that work with Arduino interface units and the open-code software available in blogs widen the application field of our proposed setup.

\section{Acknowledgments}

This work was done within the Latvian Ministry of Education and Science Council project „IMIS2“. 


\section{REFERENCES}

1. Thibos, L.N., Applegate, R.A., Schwiegerling, J.T. Standards for Reporting the Optical Aberrations of Eyes Journal of Refractive Surgery 18 2002: pp. S652-S660.

2. López-Gil, N., Martin, J., Liu, T., Bradley, A., Díaz-Muñoz, D., Thibos, L.N. Retinal Image Quality During Accommodation Ophthalmic and Physiological Optics 33 2013: pp. 497-507. http://dx.doi.org/10.1111/opo.12075

3. Van Den Berg, T.J.T.P., $\quad$ Franssen, L., Coppens, J.E. Straylight in the Human Eye: Testing Objectivity and Optical Character of the Psychophysical Measurement Ophthalmic and Physiological Optics $29(3)$ 2009: pp. $345-350$. http://dx.doi.org/10.1111/j.1475-1313.2009.00638.x

4. Bueno, J.M., Berrio, E., Ozolinsh, M., Artal, P. Degree of Polarization as an Objective Method of Estimating Eye Scattering Journal Optical Society of America A21 2004: pp. $1316-1321$.

5. Donnelly, W.J. The effects of Scatter on the Measurement of the Aberrations Materials of the 6th International Wavefront Congress 2005: pp. $21-22$.

http://voi.opt.uh.edu/voi/WavefrontCongress/2005/presentati ons/19-Donnelly-Scatter.pdf; retrieved 12.07.2015.

6. Bach, M., Mathieu, M. Different Effect of Dioptric Defocus Vs. Light Scatter on the Pattern Electroretinogram (PERG) Documenta Ophthalmologica 108 2004: pp. $99-106$. http://dx.doi.org/10.1023/B:DOOP.0000018415.00285.56

7. Barrionuevo, P., Colombo, E., Corregidor, D., Jaén, M., Issolio, L. Evaluation of the Intraocular Scattering Through Brightness Reduction by Glare Using External Diffusers to Simulante Cataracts Optica Applicata 40 (1) 2010: pp. $63-75$.

8. Kuan, M.C., Hongwen, R., Shin, T.W. PDLC-based VOA with A Small Polarization Dependent Loss Optics Communications 282 (22) 2009: pp. 4374-4377. http://dx.doi.org/10.1016/j.optcom.2009.08.012

9. Glass Apps, Electrochromic Glass vs. PDLC - Smart Glass Technology Comparison. http://www.glassapps.com/electrochromic-glass; retrieved 12.07.2015.

10. Ozolinsh, M. Dynamic Behavior of Electro-Optic and Strain-Optic Effects in Ferroelectrics with Diffuse Phase
Transitions Materials Research Bulletins 1982: pp. $741-748$.

11. Gabay, C., Berge, B., Dovlillaire, G., Bucourt, S. Dynamic Study of a Varioptic Variable Focal Lens

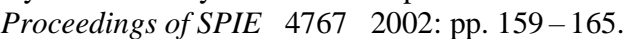

12. Ozolinsh, M., Stock, K., Hibst, R., Steiner, R., Meijer, T. PLZT Ceramics Electrooptic Modulators for Solid state $\mathrm{Nd}: Y A G$ and Er:YAG Lasers IEEE International Symposium on Applications of Ferroelectrics 1 1996: pp. $177-180$.

13. Ozolinsh, M., Hanstorp, D., Lagerwall, S.T. Optical Bistability in PLZT Ceramics Ferroelectrics 201 (1) 1997: pp. $295-304$.

14. Ramanitra, H., Chanclou, P., Dupont, L., Vinouze, B. Polymer-dispersed Liquid Crystal Structure for Variable Optical Attenuator Application Optical Engineering 43 (6) 2004: pp. $1445-1453$.

15. Ozolinsh, M., Papelba, G. Eye Cataract Simulation Using Polymer Dispersed Liquid Crystal Scattering Obstacles Ferroelectrics 304 2005: pp. 207-212.

16. Bueno, J.M., Ozolinsh, M., Ikaunieks, G. Scattering and Depolarization in a. Polymer Dispersed Liquid Crystal Cell Ferroelectrics 370 2008: pp. 18-28.

17. Chang, M.H., Das, D., Varde, P.V., Pecht, M. Light Emitting Diodes Reliability Review Microelectronics Reliability 52 2012: pp. 762-782. http://dx.doi.org/10.1016/j.microrel.2011.07.063

18. O'Brien, B., Norby, G., Li, G., Li, J. High Efficiency White Organic Light Emitting Diodes Employing Blue and Red Platinum Emitters Journal of Photonics for Energy 4 (1) 2014: pp. 043597/1 - 8

19. Banzi, M., Shiloh M. Getting Started with Arduino, 3rd Edition O'Reilly Media 2011: p. 262.

20. Monoyios, K., Perkins, J. Gamma and White Point Explained: How to Calibrate Your Monitor Scientific American Blog 17 (1) 2012: pp. 1 - 17. http://blogs.scientificamerican.com/symbiartic/how-tocalibrate-your-monitor/; retrieved 12.07.2015

21. Ozolinsh M., Eichler, H.J., Liu, B., Zhu, Q. PLZT Electrooptic Q-switch for Er: Cr: YSGG laser Proceedings of SPIE 3863 1999: pp. 521-527. http://dx.doi.org/10.1117/12.364363 